\title{
Dealing with low-incidence serious diseases in general practice
}

\author{
Frank Buntinx, David Mant, Ann Van den Bruel, Norbert Donner-Banzhof and Geert-Jan Dinant
}

\begin{abstract}
Cost-effective health care depends on high-quality triage. The most challenging aspect of triage, which GPs confront on a regular basis, is diagnosing rare but serious disease. Failure to shoulder any risk in this situation overloads the health system and subjects patients to unnecessary investigation. Adopting too high a risk threshold leads to missed cases, late diagnosis, and sometimes avoidable death. It also undermines the credibility of primary care practitioners. Quantification of diagnostic risk suggests there is a potential risk gap between the maximum certainty with which GPs can assess the risk of serious disease at presentation and the minimum certainty required by many health systems for further investigation or hospital referral. Physician gutfeeling and diagnostic safety netting are often employed to fill the gap. Neither strategy is well defined or well supported by evidence. It should be possible to reduce the diagnostic risk gap cost-effectively by adopting more explicit diagnostic algorithms and providing better GP access to new diagnostic technologies. It is also essential, given the decreasing experience of triage clinicians employed in a number of countries, that a teachable evidence base is constructed for gut feeling and diagnostic safety netting. However, this construction of an evidence base requires very large-scale studies, and the global primary care research community remains small. The challenge therefore needs to be met by urgent and effective international collaboration.

\section{Keywords}

diagnoses and examinations; diagnostic techniques and procedures; gut feeling; low-incidence diseases; safety netting.
\end{abstract}

\section{THE CLINICAL PROBLEM}

On a more-or-less regular basis, GPs have to deal with situations in which there is a real, but very low, likelihood of a serious disease. For example, while most ill-appearing children seen in general practice have self-limiting infections, about 1 in 200 of them will have life-threatening sepsis or meningitis. ${ }^{1}$ Similarly, about 1 in 200 adults who present to their GP with a persistent cough will have lung cancer, ${ }^{2}$ and 1 in 350 patients with low-back will have a serious underlying disorder. ${ }^{3}$ Serious disease in adults presenting with chest pain is a little more common, but still 19 out of 20 such patients do not have serious cardiac or respiratory problems. ${ }^{4}$

Sometimes, the patient's clinical presentation is sufficiently clear to make the GP realise immediately that there is a serious problem, whether or not it is immediately possible to make a clear-cut diagnosis. In such cases GPs will refer their patients for further investigation and treatment in hospital. The situation is serious for the patient, but for the GP there is no real diagnostic problem. The difficulty for the GP is that more often the initial clinical picture is

F Buntinx, MD, PhD, professor and research director, Department of General Practice, Catholic University of Leuven, Belgium and Department of General Practice, University of Maastricht, the Netherlands. D Mant, FRCGP, FRCP, professor of general practice and head of department, Primary Health Care; A Van den Bruel, MD, PhD, academic clinical lecturer, Department of General Practice, University of Oxford, Oxford. N Donner-Banzhoff, MD, PhD, professor and head of department, Department of General Practice, University of Marburg, Marburg, Germany. G-J Dinant, PhD, $M D$, professor of clinical research in general practice, Department of General Practice, University of Maastricht, Maastrict, the Netherlands.

Address for correspondence

Professor Frank Buntinx, Katholieke Universiteit Leuven Department of General Practice, Kapucijnenvoer 33, blok J, bus 7001, Leuven, 3000, Belgium.

E-mail: frank.buntinx@med.kuleuven.be

Submitted: 12 February 2010; Editor's response: 24 February 2010; final acceptance: 24 May 2010.

OBritish Journal of General Practice 2011; 61: 43-46. 


\section{How this fits in}

GPs have to deal with situations in which there is real, but very low, likelihood of disease. Gut feeling, simple heuristics, and safety netting methods are being used in such situations, but there is insufficient evidence for underpinning the optimal use of such methods. Building better algorithms and introducing new technological tests, together with further studying of teachable gut feeling content and optimal safety netting techniques are urgently needed. This research will need large scale investigation by an effective international collaboration, firmly based in everyday general practice.
- arranging investigations to which they have access; and

- applying diagnostic safety netting.

The problem is that the evidence base underpinning these four strategies is poor. Moreover, in a number of countries, diagnostic triage is increasingly being carried out by less and less experienced clinicians.

\section{GUT FEELING}

Gut feeling, the feeling that something serious is wrong, is reported by GPs to be an important diagnostic tool in many European countries. ${ }^{8,9}$ It was found to be the most discriminative 'test' in diagnosing adult patients with chest pain, ${ }^{4}$ or ill children. ${ }^{1}$ Nobody suggests that gut feeling is a special paranormal gift of GPs, ${ }^{10}$ although the finding could not be confirmed in patients with chest pain examined in a university teaching hospital's emergency department. ${ }^{11}$ Most likely, GPs combine a number of verbal and non-verbal clues, however it is not possble to disentangle this information or the way in which it is used. However, while GPs continue to allow gut feeling to remain a poorly described mystic state gained only by experience, we cannot teach the use of this information to students or young GPs. If it really is dependent on experience, we need to define exactly what the necessary seminal experiences are so that we can ensure that they are included in GP training. This requires research on the components of gut feeling and its diagnostic value. Such research has to be performed in emergency situations, but also in routine consultations where low-prevalence serious diseases like cancer can be diagnosed..$^{12}$

\section{DIAGNOSTIC ALGORITHMS}

GPs have a history of using heuristics and simple rules that are adapted to their practice environment. ${ }^{13,14}$ They aggregate individual bits of diagnostic information (often each of low diagnostic value) to achieve a composite diagnostic decision of high sensitivity. ${ }^{15}$ Subgroups with a higher or lower likelihood of disease can be based on the specific epidemiological information collected in a general practice setting. Below the age of 50 years, rectal bleeding almost never refers to colorectal cancer, while the likelihood of malignancy rises to one in five in old age. ${ }^{16}$ With slowly developing illnesses like cancer, it is possible to collect this diagnostic information sequentially over a number of days.

However, the problem remains that for all serious diseases any algorithm applied must achieve high sensitivity - particularly to avoid missing dangerous rapidly progressive illness such as sepsis in children. 
While it may be possible to build an algorithm to achieve this high sensitivity, it is often difficult to do this with an adequate positive predictive value (that is, without causing unnecessary hospital admissions and anxiety to patients or parents). For example, a simple diagnostic algorithm based on five presenting clinical features achieves very high sensitivity in diagnosing serious infection in children. ${ }^{1}$ However, this high sensitivity is achieved only by unacceptably low predictive value - the algorithm identifies $12 \%$ of children as potentially at risk, most of whom will have non-serious illness.

\section{INVESTIGATIONS AND MEASUREMENTS}

GP access to diagnostic investigations varies between health systems. Technological advance is tending to increase this access, reducing the cost, and making point-of-care testing feasible. For example, a key observation arising from the meningococcal research was that about half of the children missed had symptoms that suggested that vital signs (breathing and heart rate) may well have been abnormal if measured. ${ }^{7}$ New technology is already making it cheap and simple to measure pulse and oxygen saturation in general practice; it may soon be possible also to measure breathing rate using the same pulse oximeter technology..$^{12,17}$ Others have suggested diagnostic benefit from the use of near-patient laboratory tests in general practice such as $\mathrm{C}$-reactive protein or procalcitonin. ${ }^{18}$ However, any diagnostic technology that is going to be used in routine practice has not only to be robust but also to comply with minimal requirements with respect to volume and weight. Test results have to be available within a couple of minutes, and the price must be modest. The chances are high that the development of cost-effective diagnostic technologies to reduce the diagnostic risk gap will require many years of multidisciplinary developmental and evaluation work, and a lot of money.

\section{SAFETY NETTING}

Even if a patient is considered to give no reason for alarm, things can still go wrong. The GP therefore has to put a safety net in place. ${ }^{19,20}$ As recently described by Almond et al, clearly communicating to patients or parents what exactly you know and don't know (and thus what could happen subsequently) is an important initial step; ${ }^{19}$ this should be followed by clear information and advice on re-contacting the GP in specific situations. Good GPs will always do this, but we have no data on the frequency and methods of use, or evidence on the relative advantages and disadvantages of different approaches. Many colleges and organisations have drawn up written safety-net advice to give to patients and parents in a number of situations. However, in the absence of a firm evidence base, their content varies and many raise practical questions about how they should be used in the consultation. What should you say to a patient when giving the written leaflet? When will you ask them to re-consult? Systematically calling back a patient (for example, an ill child) after a couple of hours, or asking patients to call back their GP may be an effective option, but if the first contact takes place at the end of the day, neither the patients nor the GP on duty will be pleased with advice to perform such calls if all remains well. Safety-netting strategies and subsequent action (whether referral, additional testing, or reassuring parents) ought to be evaluated on their effect. What is the effect on patient outcome? What is the effect on appropriate and inappropriate referral rates? What is their effect on antibiotic prescribing? What is the effect on parental anxiety and distress? Large-scale research will be needed to answer these questions.

\section{THE PROBLEM OF SCALE}

The problem facing researchers trying to build an effective evidence base for an optimal diagnosis of rare disease is largely one of scale. For example, the study by Van den Bruel et al required more than 100 clinicians to recruit 4000 ill children but still had limited statistical power as only 31 children with serious diseases were identified (nine of whom had meningitis or sepsis). ${ }^{5}$ The power of multivariate analyses performed on the resulting database can only be less than optimal. Individual practitioners like John Fry have in the past conducted large-scale studies in a single practice by collating data on the presenting features of all their patients over many years, but this is difficult to replicate with the constraints of modern practice. Although computerisation has made the collection and analysis of routine data much easier, exemplified by the UK General Practice Research Database, ${ }^{21}$ in most cases we know that the routine data collected do not reliably include the detail about presenting symptoms necessary for high-quality diagnostic research. It may be feasible to set up a database study in selected practices to prompt collection of more detailed diagnostic information, but the necessary quality control of data and validation of diagnoses still makes the time and finance required to conduct a diagnostic study of a rare disease very difficult to achieve on a national scale.

\section{TAKING THINGS FORWARD}

The current approach to diagnosing rare disease is unsustainable. The general public is becoming less tolerant of risk taken on their behalf, and we risk our professional credibility if we do not address the 
diagnostic risk gap. Across Europe, changing working practices mean that the individuals conducting primary care diagnostic triage are becoming less experienced and less familiar with the patient. Reliable gut feeling requires experience and knowledge of the patient. We can improve diagnosis cost-effectively by adopting more explicit diagnostic algorithms and by providing better GP access to new diagnostic technologies. It is also essential that we construct a teachable evidence base for gut feeling, ${ }^{22}$ and diagnostic safety netting. ${ }^{19}$ The urgent challenge is to provide young doctors and other clinicians with better evidence-based training and diagnostic tools for triage, especially at registrar/vocational training level.

However, this construction of an evidence base requires very large-scale research and the global primary care research community remains small. It will be difficult for researchers in any one country to marshal the necessary resources for a series of diagnostic studies that require a sample size of 100000 or more. The challenge therefore needs to be met by urgent and effective international collaboration. All these issues call for the establishment of an international collaborative group devoted to improvement of the approach towards patients with a possible low-incident serious disease in general practice. However, this is not just a task for general practice researchers - the challenge extends to physicians working in emergency departments, biostatisticians, and industry who need to help develop and assess the diagnostic benefits of emerging technology. And above all, the research evidence needs to be collected from everyday general practice, including the active research involvement of service GPs on an unprecedented scale. The authors' recent experience suggests this is possible.

\section{Acknowledgements}

We are grateful to Bert Aertgeerts, Jan Verbakel, Matthew Thompson, Susanna Almond, Anthony Harnden, and Rudi Bruyninckx for their comments on a previous version of this text and to the members of the Maastricht Mesch group for an excellent meal and a very lively discussion.

\section{Competing interests}

The authors have stated that there are none.

\section{Discuss this article}

Contribute and read comments about this article on the Discussion Forum: http://www.rcgp.org.uk/bjgp-discuss

\section{REFERENCES}

1. Van den Bruel A, Aertgeerts B, Bruyninckx R, et al. Signs and symptoms for diagnosis of serious infections in children: a prospective study in primary care. Br J Gen Pract 2007; 57(540): 538-546.

2. Hamilton W, Peters T, Round A, Sharp D. What are the clinical features of lung cancer before the diagnosis is made? Thorax 2005; 60(12): 1059-1065.

3. Donner-Banzhoff N, Roth T, Sönnichsen AC, et al. Evaluating the accuracy of a simple heuristic to identify serious causes of low back pain. Fam Pract 2006; 23(6): 682-686.

4. Buntinx F, Truyen J, Embrechts P, et al. Evaluating patients with chest pain using classification and regression trees. Fam Pract 1992; 9(2): 149-153.

5. Van den Bruel A, Haj-Hassan T, Thompson M, et al. Diagnostic value of clinical features at presentation to identify serious infection in children in developed countries: a systematic review. Lancet 2010; 375(9717): 834-845.

6. Thomson CS, Forman D. Cancer survival in England and the influence of early diagnosis: what can we learn from recent EUROCARE results? Br J Cancer 2009; 101(suppl 2): S102-109.

7. Thompson MJ, Ninis N, Perera R, et al. Clinical recognition of meningococcal disease in children and adolescents. Lancet 2006; 367(9508): 397-403

8. Stolper E, Van Royen P, Van de Wiel M, et al. Consensus on gut feelings in general practice. BMC Fam Pract 2009; 10: 66.

9. Stolper E, van Royen P, Dinant GJ. The 'sense of alarm' ('gut feeling') in clinical practice. A survey among European general practitioners on recognition and expression. Eur J Gen Pract 2010; 16(2): 72-74.

10. Stolper E, van Bokhoven M, Houben P, et al. The diagnostic role of gut feelings in general practice. A focus group study of the concept and its determinants. BMC Fam Pract 2009; 10: 17.

11. Knockeart DC, Buntix F, Stoens N, et al. Chest pain in the emergency department: the broad spectrum of causes. Eur J Emergency Med 2002; 9: 25-30.

12. Hamilton W, Stange KC. A nose for cancer: clinicians' clinical intuition and the diagnosis of cancer. International Primary Care and Cancer Research Group Conference, 23 and 24 April 2009, Copenhagen, Denmark. Book of abstracts 2009; abstract 4. http://folkesundhed.au.dk/fileadmin/www.folkesundhed.au.dk//forsk ningsenheden_for_almen_praksis/cancer_research_group/presentati ons/boa.pdf (accessed 30 Nov 2010).

13. Henaghan C, Glasziou P, Thompson M, et al. Diagnostic strategies used in primary care. BMJ 2009; 338: b946.

14. Andre M, Borgquist L, Foldevi M, Mölstad S. Asking for 'rules of thumb': a way to discover tacit knowledge in general practice. Fam Pract 2002; 19(6): 617-622.

15. Buntinx F, Aertgeerts B, Aerts M, et al. Multivariable analysis in diagnostic accuracy studies. What are the possibilities? In: Knottnerus A, Buntinx F (eds). The evidence base of clinical diagnosis - theory and methods of diagnostic research (BMJ books). Oxford: Blackwell Publishing Ltd, 2008: 146-166.

16. Wouters H, Van Casteren V, Buntinx F. Rectal bleeding and colorectal cancer in general practice: diagnostic study. BMJ 2000; 321(7267): 998-999.

17. Fleming S, Tarassenko L, Thompson M, Mant D. Non-invasive measurement of respiratory rate in children using the photoplethysmogram. Conf Proc IEEE Eng Med Biol Soc 2008; 2008: 1886-1889.

18. Cals JW, Butler CC, Hopstaken RM, et al. Effect of point of care testing for $\mathrm{C}$ reactive protein and training in communication skills on antibiotic use in lower respiratory tract infections: cluster randomised trial. BMJ 2009; 338: b1374.

19. Almond S, Mant D, Thompson M. Diagnostic safety-netting. $\mathrm{Br} J$ Gen Pract 2009; 59(568): 872-874.

20. Neighbour R. The inner consultation. Oxford: Radcliffe Publishing, 2004.

21. Jones R, Latinovic R, Charlton J, Gulliford MC. Alarm symptoms in early diagnosis of cancer in primary care: cohort study using General Practice Research Database. BMJ 2007; 334(7602): 1013-1014.

22. Stolper E, van Leeuwen Y, van Royen P, et al. Establishing a European research agenda on 'gut feelings' in general practice. A qualitative study using the nominal group technique. Eur J Gen Pract 2010; 16(2): 75-79. 